\title{
Modelo multidireccional para la generación de innovación educativa
}

\author{
Rodrigo Urcid Puga ${ }^{1 a}$ y Juan-Carlos Rojas ${ }^{2 b}$
}

\author{
Tecnologico de Monterrey, Escuela de Humanidades y Educación ${ }^{a}$ \\ Tecnologico de Monterrey, Escuela de Arquitectura, Arte y Diseñob
}

ORCID ID: https://orcid.org/0000-0002-5102-35581

ORCID ID: https://orcid.org/0000-0001-7718-4555²

\section{Recibido: 11 de mayo 2018}

Aceptado: 28 de agosto de 2018

\section{Resumen}

El siguiente artículo tiene por objetivo presentar un modelo que refleja la necesidad de innovación educativa que las Instituciones de Educación Superior (IES) deben considerar al momento de replantear su administración, renovación de planes educativos, perfiles de ingreso y egreso y la colaboración con los diferentes grupos de interés de la industria y sociedad. La metodología de este estudio es cualitativa, y se sustenta en una investigación documentada y de corte transversal. Como resultado, se muestra un modelo que representa los diversos elementos que componen la necesidad y pertinencia de la innovación educativa en las IES. A través del modelo elaborado, se puede comprobar que la innovación educativa es una realidad actual, y que todas las IES deben asumir sus capacidades para darle la importancia y tomar acciones de cambio.

Palabras Clave: Política educativa, organización de la universidad, administración educativa, paradigmas en la educación.

\footnotetext{
${ }^{1}$ Correspondencia al autor

E-mail: rurcid@itesm.mx
} 


\title{
Multidirectional model to generate educational innovation
}

\begin{abstract}
This article aims to present a model that reflects the need for educational innovation that higher education institutions (HEIs) should consider when restructuring their administration, renewing their educational curriculum including entrant/graduate profiles, and collaborating with the various interest groups within the industry and society. The methodology of this study is qualitative, supported by a documented and cross-sectional investigation. As a result, a model is shown that represents the various elements that make up the need and relevance of educational innovation in HEIs. Through this model, educational innovation is shown to be a current necessity and all HEIs must assume their capacities to give it importance and take action for change.
\end{abstract}

Key-words: Educational policy, university organization, educational administration, paradigms in education.

\section{Introducción}

La sociedad se transforma constantemente, y por eso, las necesidades son diferentes. Como consecuencia de lo anterior, las IES o universidades juegan un papel importante para definir un estatus e ideologías que las caracterizan. Sumado a esto, a las universidades se les define como motores de desarrollo científico y cultural en las ciudades y estados; por ello, estos espacios hacen que el alumnado tenga intereses cada vez más diversos y más especializados, el mercado laboral sea más exigente, y la innovación sea indispensable (Tójar y Matas, 2005).

Por otra parte, en los últimos años, la educación en las IES se ha visto modificada de manera considerable, de hecho, ha sido tal el cambio, que los estudiantes se han vuelto partícipes de su propio aprendizaje, concretamente, se convierten en alumnos y alumnas capaces de aprender de forma autónoma y autorregulada; es decir, tienen la capacidad de gestionar los espacios físicos en los cuáles han de trabajar, los tiempos -horarios- en los que consideran oportuno incrementar su conocimiento y las herramientas tecnológicas necesarias para acompañar los diversos procesos de aprendizaje. Por ello, es necesario "aprender a aprender", pues este proceso se manifiesta durante toda la vida, pero que se gesta particularmente durante la etapa de la educación académica (Malander, 2014).

Del mismo modo, las IES no deben estar al margen de la sociedad, pues se enfrentan a diversas vicisitudes como el económico, la igualdad de condiciones de acceso, el profesorado con diversas 
habilidades y capacitaciones, las Tecnologías de la Información y Comunicación (TICs), el pensamiento disruptivo, entre otras. A la par, el mercado laboral ahora tiene otras características, mismas que son diferentes a las de hace algunos años y, en particular, se logra un sentido de lo educativo, lo social, la investigación y los valores (Álvarez y Lázaro, 2002).

En relación con lo anterior, Pedroza, García y Briceño (2005), sostienen que las IES realizan modificaciones en las estructuras académicas y administrativas con base en el entendimiento de que fijar la innovación se despliega a la economía del conocimiento; todo esto, adquiere más importancia para la economía como un bien intercambiable para la libre circulación de conocimiento científico y tecnológico. En cuanto a las organizaciones, al igual que en las sociedades, no pueden darse cambios de estructura sin cambios de función. En una organización amplia donde el todo permite a las partes desempeñar acciones independientes, dicha interacción puede propiciar una unión tan fuerte, que, si es separada, puede traer graves consecuencias (García, 2006).

En la dinámica de la generación de nuevo conocimiento, los indicadores se convierten factores que sirven como aproximación al entendimiento de su dinámica, la aplicación práctica y pertinencia para el desarrollo de la ciencia y su comunidad científica (Ramírez, 2005). Implícito en la generación de nuevo conocimiento, la innovación per se, consiste un cúmulo de acciones planificadas las cuales incluyen un cambio perseguido, planificado y desarrollado con intencionalidad. Por su lado, se dice que el cambio consiste en una serie de procesos de aprendizaje (Fullan, 1996). Así pues, y al tomar a las IES como elemento susceptible a la innovación, el objetivo es mejorar la organización con una meta tangible; y que por supuesto, el impacto al alumnado y los espacios para ayudar a su formación (Marcelo, 1996).

Actualmente, los sistemas educativos se enfrentan a una contradicción constante que va de la conservación a la creación contradictoria para algunos, y complementaria para otros. Es decir, de acuerdo con el propio concepto de educación, los sistemas relacionados con la misma, tienen doble asunto: conservadora-reproductora y con la capacidad de cambiar como necesidad social para mejorar a los individuos (García, 2006); el término innovación en educación, suele ser más utilizado; el verbo innovar, conlleva el logro de cambios trascendentes que deriven en mejoras.

De manera general el proceso de innovación se caracteriza por tener una secuencia de acciones que inician con la detección de problemas o elementos contraproducentes, hasta la evaluación de las sugerencias propuestas. Así, y para que los resultados sean óptimos, lo ideal es hacer que los 
actores involucrados se vean inmersos en las propuestas de innovación; y es que al final del día, son ellos quienes hacen uso de estos elementos, por ello, lo más prudente es la existencia de una enseñanza acerca de la práctica y desarrollo de la innovación (Fernández, 2006).

Livingston y Assunção Flores (2017) realizan una documentación de los últimos 40 años de tendencias en educación en Europa, países referentes en el tema de innovación, donde describen más de 25 temas en los que incluyen desde la aplicación de las tecnologías hasta la educación en casa. Esto evidencia que el proceso educativo está más allá de los elementos conocidos en la educación tradicional: cursos presenciales, aulas establecidas y no dinámicas, contenidos curriculares, diseño de planes de estudio, admisión de estudiantes, entre otros aspectos (Alatorre, 2006).

Actualmente, la innovación educativa está en un proceso de derribar barreras y asociar potenciadores para transformar las IES. Algunos de estos potenciadores son la cooperación social, el empoderamiento y descentralización, la gestión acertada, nuevas estructuras organizacionales, la transparencia y la innovación centrada a la organización de la institución (Lašákováa, Bajzíkováa, y Dedze, 2017). La innovación relacionada con el ámbito educativo considera todo un sistema que contempla la implementación de productos, alternativas y constructos novedosos, así como la gestión y su impacto en el perfeccionamiento de la educación (Laurencio y Farfán, 2016).

De este modo, la innovación se vuelve un reflejo crítico y una herramienta de transformación; además, requiere una visualización de diversos factores, en particular algunas prácticas que fomenten cambios positivos en diversas funciones y áreas en materia de educación. La perspectiva de Laurencio y Farfán (2016), describe lo anterior, la gestión organizacional, la administración y la proyección pedagógica de la formación constituyen aristas básicas en el crecimiento de la innovación educativa.

Centrándonos en los procesos de enseñanza y apropiación de conocimiento que se suelen manifestar en las IES poseen elementos muy concretos que los diferencian de la educación básica y por eso, es en dicha fase cuando se ponen de manifiesto las exigencias, los cambios de comportamiento del profesorado, los cambios organizativos y las transformaciones metodológicas (Tójar y Matas, 2005).

Es necesario analizar postulados metodológicos que posibiliten gestionar de manera efectiva y eficaz las acciones que suelen afectar cuando se busca la calidad de las IES, esto, necesita investigaciones profundas y un grado de vigilancia sobre el riesgo que las universidades tienen al 
aplicar cuestiones tanto teóricas como prácticas no estandarizadas o convencionales; así como aclarar las condiciones en donde se aplican (Pons, Barrios y Puello, 2010). Un tema relacionado con la idea anterior es la interdisciplinariedad, la cual "se aboca a restaurar los vínculos entre los diversos dominios cognoscitivos para tributar a una mejor comprensión de los fenómenos" (Castro y Chávez, 2012, pp. 55). Conviene mencionar que las diversas perspectivas inter, multi, y transdisciplinarias se refieren a las "disciplinas convocadas para el tratamiento de un fenómeno determinado, más que a una verdadera integración de métodos, conceptos y lenguajes, aspecto que distingue a la perspectiva interdisciplinar de las anteriores" (Guzmán, 2009, pp. 3).

El crecimiento de la inter y de la unión entre disciplinas en el proceso de aprendizaje y desarrollo educativo actuales exige un cambio drástico en las IES, pues al tocar el tema de la educación, no se puede separar el desarrollo de la sociedad del cambio académico, a partir de lo anterior es que se define que la transdiciplinariedad, multidisciplinariedad, e interdisciplinariedad son básicas para lograr un replanteamiento de la innovación educativa en las universidades. En este sentido, es necesario considerar que el proceso de enseñanza y aprendizaje ha crecido de manera exponencial, y por ello, los estudiantes deben ser activos al momento de estar formándose, lo cual tiene por consecuencia el aprendizaje tanto autónomo como autorregulado.

Todo ello se ve fortalecido a través de una educación basada en diversas disciplinas. Dentro del contexto de las disciplinaridad, se encuentra un elemento que las universidades tiene como finalidad, la inserción de los estudiantes al mundo laboral. Hasta hace algunos años se pensaba que, si se contaba con una carrera, esto significaría que se tiene un deseo de superación personal y por supuesto, a la permanencia laboral. Sin embargo, esto ya no es así, pues la educación superior ya no se valora a nivel del profesionista, y uno de los elementos fundamentales para que esto se presente es la sobrepoblación universitaria; caso concreto son las universidades públicas mexicanas, las cuales están ampliamente rebasadas por la demanda estudiantil la cual está en constante crecimiento.

Sin embargo, existe otra tendencia que dice que las IES deben valorar la reducción de la matrícula para poder enfocarse en un nuevo tipo de enseñanza basada en centrarse en el estudiante. Los problemas de la sobrepoblación el cambio de enfoque y el desinterés en las universidades consiguen crear profesionistas que realizan labores diametralmente ajenas a su formación (Téllez, 2011). Finalmente, para cerrar esta introducción el modelo que se presenta más adelante, debe 
considerar la intervención de las instituciones de gobierno, regionales e incluso las internacionales para poder potenciar la innovación en las IES (Beaver, 2001).

\section{Marco teórico}

La finalidad de este artículo es exponer un modelo que replantee el uso de la innovación en las IES. Esta es la idea primaria que se ha explicado introductoriamente en la primera parte del artículo. Sin embargo, hay otros tantos elementos que son de vital importante para tener un mejor entendimiento del modelo que se presenta. En los párrafos venideros se hace mención de varios factores como la educación trans, multi e interdisciplinar, el marco contextual, el cambio o adaptación de la enseñanza y el impacto de esta. Además de introducir los elementos que conectan estos factores: el lean thinking y la apertura al cambio.

En términos generales, cualquier modelo de innovación educativa tiene que considerar algunos aspectos elementales de la enseñanza de las IES: aspectos científicos, tecnológicos, socioculturales y actividades disruptivas. Estos elementos son fundamentales pues suelen ayudar a la mejora de cualquier organización, incluso a países desde diversas aristas. Un ítem que debe contemplarse es la innovación, existen una serie de análisis y estudios que lo colocan como un actor elemental en los distintos puntos que suelen regir a una nación, tal es el caso del rubro económico o social; todo ello, en lo que ahora se conoce como la economía del conocimiento (Alemán, Gómez, Parada y Sainz, 2012).

Para este modelo la innovación forma parte de la filosofía que conecta los factores, en el trabajo de Santiago y Andía (2016) se menciona a la innovación como "un camino mediante el cual el conocimiento se traslada y se convierte en un proceso, un producto o un servicio que incorpora nuevas ventajas para el mercado o para la sociedad (Formichella, 2005, pp. 4)”.

Además, se comparte con Edgar y Grant (2009) en Alemán, Gómez, Parada y Sainz, (2012), a la innovación como un aspecto esencial para impulsar elementos a un nivel superior; también mencionan que ésta se configura como el saber que ayuda a conformar el entorno y la posibilidad de un cambio por parte de ellos. Al término innovación se le puede concebir, como un "camino mediante el cual, el conocimiento se traslada y se convierte en un proceso, un producto o un servicio que incorpora nuevas ventajas para el mercado o para la sociedad" (Formichella, 2005, p. 4). Esta concepción extiende desde la innovación en productos, hasta en procesos y organizaciones (Tourón, 2014). 
Al referirse al tema de innovación hay que incluir una relación con conceptos como cambio y mejora que, en diversas situaciones, se utilizan como sinónimos y con un vínculo estrecho. Otro aspecto elemental en la definición de innovación es la evaluación y ésta sólo puede valorarse en relación con las metas y objetivos predeterminados; de ahí que una de sus principales características es ser un proceso (Rodríguez, 2015). Al considerar lo antes mencionado, es posible hacer un nexo con la innovación educativa, la cual ya no sólo es una oportunidad de mejora para las IES, ahora, es un factor necesario para el cumplimiento de las metas en aras de la competitividad, vista, claro, desde una perspectiva de innovación educativa (Ortega et al., 2007; López, 2010).

Así, en relación con la educación e innovación, se tienen significados y particularidades propias del contenido y dinámica en la cual se registra, sin embargo, las que aquí se enuncian se consideran las primarias: a) dirigen y encaminan; b) buscan perfeccionar la mayor cantidad de habilidades intelectuales; c) afinan y perfeccionan los sentidos; y, d) ayudan a fomentar valores morales (Rodríguez, 2015).

Sin embargo, sin importar cualquiera de las características mencionadas se puede hablar de que el propósito de la educación es reorganizar la subjetividad de aquellos quienes la reciben. También, la innovación en educación tiene un reto en particular, el proceso de adopción, mismo que debe ser "abrazado" por diversos grupos de interés, y que, a diferencia de las cosas materiales, modificar las actitudes, prácticas y valores humanos es más complejo (Rodríguez, 2015).

Así, a nivel educativo, existen tres factores fundamentales y con ello tres estilos de interacción: el primero se refiere al educador (profesor) quien es el responsable de conducir la modificación de la subjetividad, el segundo es la persona que aprende (alumno); quien se convierte en un elemento activo en la transformación de su propia subjetividad y en la formación cultural.

Por último, está el conocimiento o currículum, mismo que se convierte en contenido para ser adquirido; Silas (2000-2001) en Silas (2006). Reforma, innovación, cambio y renovación son términos que actualmente se emplean de manera continua, lo cual ha ocasionado la pérdida de su potencial; en ocasiones se utilizan como la utopía a nivel semántico, aunque su denominación responde al alcance, profundidad o trascendencia de las acciones realizadas (Alatorre, 2006).

Los cambios que conllevan implementar la innovación educativa pueden ser observados desde distintas aristas. Una forma de concebirlo es como un "proceso socioeducativo orientado a la proyección de entornos, procesos, funciones y actores educativos, con la generación, producción 
e implementación de novedades científicas y tecnológicas, en función del perfeccionamiento integral de la educación” (Laurencio y Farfán, 2016, pp. 19). Para Rodríguez-Flores (1997), en García, (2006), el cambio a nivel educativo se puede traducir como la transformación o modificación que tienen lugar en los conceptos educativos y en la capacidad para ponerlos en marcha, incluyendo los que emanen de la instrumentación práctica. Lo cual conlleva a algunos principios para llevarlos a cabo en las organizaciones de educación:

- Participación continua.

- Criterios con las actividades realizadas.

- Motivación.

- Desarrollar y estimular la creatividad.

Tener una idea diferente en cuanto a los aspectos educativos, conlleva a mencionar áreas específicas de la vida cotidiana, o en su caso, inciden en los elementos esenciales de la institución escolar (García, 2006). Al seguir con la temática del cambio educativo, la acción o efecto de introducir novedades para alterar las cosas, se le conoce como innovación.

Del mismo modo, a la innovación, se le puede definir como la "puesta en práctica de algo nuevo que hace frente de mejor manera a las condiciones del entorno; esta nueva forma de acoplarse necesita un análisis de las condiciones y una nueva puesta en marcha de una solución” (Silas, 2006, pp. 36). Esta solución o propuesta parte de un cambio en el estilo en que individuos o las organizaciones suelen actuar; es decir, tienen la capacidad de modificarse y acoplarse con el entorno que les rodea, es decir, evolucionar. Se ha trabajado mucho en entender los conceptos de cambio, innovación y mejoras en las IES, por ello, Latinoamérica debe preparse para adoptar, mejorar y diseñar su filosofía para la enseñanza.

Para Ezpeleta (2004), la innovación puede ser algo nuevo que es percibido como tal por la comunidad, por lo que puede serlo o no; esto lleva a la discusión sobre lo que es la generación, investigación y validación, la propia innovación educativa y quiénes son los que referentes en la región. Sin duda, la zona latinoamericana puede generar mejor educación; México, particularmente, es de interés para la mayoría de la literatura y seminarios, congresos y talleres.

Si bien aparece un interés común, la conceptualización aún está lejos (Morales, 2006); y en este sentido, es prudente contemplar lo que Alatorre (2006), aporta en materia de educación, dicho autor comenta que los cambios son constantes, algunos leves y, en ocasiones, poco perceptibles; 
pero si éstos son trascendentes y se enfocan en la mejora, entonces se puede referir como innovaciones.

México, entro otros países del continente americano empiezan a tener una trasferencia sociocultural, un cambio de actitud, mentalidad y metodológico. Actualmente, "muchas modificaciones pueden darse para perpetuar o legitimar algo dado, aunque esto esté superado, por tanto, si se quiere hablar de innovación no sólo se tiene que apuntar hacia el cambio, sino hacia la transformación real tendiente a la superación” (Alatorre, 2006, pp. 170).

Los primeros cambios que se han presentado en las IES que pueden hacer referencia al cambio, pero no necesariamente a una mejora o innovación educativa, se refieren a la modificación en materia curricular. Se brinda especial atención a la creación de contenido académico, es decir, a los planes de estudios.

Así pues, en una sociedad globalizada como en la que actualmente se vive, demanda que las IES tengan una participación más activa, que piensen en nuevas estructuras curriculares, que tomen en cuenta el aprendizaje basado en competencias y la resolución adecuada de los retos que los profesores han de plantearles al inicio de cada ciclo escolar; todo claro, con instrumentos de evaluación declarados desde un principio (Alemán, 2010). Desde hace un par de años atrás, Pineda (1990), dice que la innovación educativa es una categoría superior, algo nuevo, pero se diferencia con respecto a quien se dirige. Más tarde, Rivas (2000), asegura que el término de innovación tiene matices en función del contexto en que se utiliza. Parece ser que en México se cuenta con historia de innovación, cosa que lleva a ver grandes cambios o mejoras (Morales, 2006).

Recientemente, Rodríguez (2015), menciona que ante un escenario donde las IES en México son tan diversas, se le exige al Estado asumir la modernización como estrategia para reordenar el crecimiento y desarrollo educativo para poder hacer frente a las realidades del país. Así, el sistema educativo ha propuesto, por un lado, cambios estructurales, reformas institucionales, innovaciones educativas; y por otro, la construcción de mejores condiciones de financiamiento, inversión y atención, esto bajo el precepto de que, al lograr la excelencia educativa en las IES, se mejora el nivel de vida de quienes habitan la región. Al retomar el tema del involucramiento que la sociedad sostiene en materia de innovación, es importante mencionar que ya existen algunas instituciones que optan por crear algunas acciones para promover la innovación; un ejemplo de estas instituciones es la Universidad Privada de Santa Cruz (ubicada en Bolivia), que destaca una serie de acciones: 
- Exposiciones de emprendimientos. Crear un espacio para que los estudiantes de las IES expongan los trabajos que han realizado a lo largo de los semestres.

- Maratones de tecnología. El alumnado enfocado en áreas tecnológicas desarrolla propuestas que den solución a inconvenientes de la vida diaria.

- Conferencias y ponencias. Alumnos y profesorado, son partícipes en este evento; mismo que tienen una temática en común. El objetivo, a través de diferentes ponencias, conferencias magistrales, talleres, y demás eventos, es motivar las ideas innovadoras y/o creativas del estudiantado.

- Propuestas de negocios. Tiene la finalidad de mostrarle a la comunidad ideas de validadas para encontrar socios estratégico o inversionistas.

- Consultoría a empresas y organizaciones privadas y/o públicas. El objetivo principal es brindar consultoría y capacitación al sector empresarial.

- Incubadora de negocios. Se busca que el estudiante desarrolle su creatividad, innovación y el emprendimiento de estudiantes y egresados de la universidad.

- Centros de investigación. La idea es desarrollar especialistas en áreas de industrialización, investigación y en la asistencia en temas relacionados con las TIC.

- Desarrolladora de empresas. Propone impulsar a los estudiantes con sentido emprendedor en aras del progreso.

- Centros de innovación. Se concibe como un centro de asesoramiento y educación continua para las organizaciones de cualquier índole.

Todos estos elementos y muchos más pueden ser implementados por las IES en vías de crear medios de innovación. Sumado a esto, Tejada (2008), comenta que toda innovación es una acción premeditada y que las experiencias deben ser contempladas con total seriedad. Mientras que Havelok y Huberman (1980) en García (2006), describen tres modelos básicos, algo que se repite después en una aproximación de Niño de Guzmán (2015) en su trabajo sobre la organización cultural actual. Los tres modelos son:

- Investigación y desarrollo: se espera un cambio racional y equitativo en cualquier persona.

- Solución de problemas: el cambio se induce a partir de las motivaciones del usuario.

- Interacción social: usar mecanismos de influencia de algunas instituciones educativas en otras. 
- Estructura institucional: serie de pasos que incluye la colaboración con otros organismos, empresas, instituciones, etc., y plantea a la innovación como parte de una sociedad responsable.

En pocas palabras, todo modelo de innovación educativa se adentra en una concepción de lo institucional; por ello, es necesario adoptar las características particulares de los diversos aspectos de la organización educativa para así, asegurar que los procesos de innovación tengan el impacto necesario (García, 2006). Finalmente, se deben mencionar tres elementos que ayudan a definir a la innovación educativa, y que, a su vez, deben utilizarse para fomentar un cambio tanto al interior como al exterior en las IES; a saber (Valle, 2012, pp. 273):

1. Las instituciones educativas deben tener una estructura como tal

2. El entorno social y cultural fuerza a que las IES se sometan al cambio.

3. Alumnado como sujeto del cambio metodológico.

Por ello, para dar por sentado un proceso de innovación educativa en las IES se debe contemplar lo siguiente (Laurencio y Farfán, 2016):

- Identificar y fundamentar los requisitos, las expectativas, metas, objetivos, motivos de las instituciones.

- Ser críticos de la sociedad, determinar lo que ésta necesita y cómo se puede mejorar.

- Definir políticas que fomenten la generación la adecuada proyección de la educación en la sociedad.

- Experimentar con elementos que ayuden a que la institución tenga actividades de innovación.

- Lograr una discusión en la que todos los miembros de la comunidad estén involucrados.

- Es necesario demostrar los elementos a favor de la innovación para que las IES cumplan con sus metas.

- Buscar la implementación de estrategias que fomenten la una educación innovadora para la mejora de las IES.

- Integrar mecanismos de innovación que integren algunos resultados institucionales.

Después de describir varios de los factores que se presentan o se desarrollan en la innovación educativa y cómo están en desarrollo no solo en las IES, sino en la sociedad en general, es necesario tocar un elemento o factor que conecta y que puede mejorar este proceso de adopción o cambio: 
lean thinking Éste, apela a "una gestión global de los procesos, por medio de una estrategia enfocada en proveer el servicio de la manera más eficaz, mejorando el flujo y eliminando los desperdicios de los procesos" (Carrillo, Pons, Barrios y Puello, 2010, pp. 2).

Lean en general, es una filosofía que tiene como meta la eliminación de cambios en los procesos para así añadir valor. Patño (2008), en Carrillo, Pons, Barrios y Puello, (2010) define lean thinking como una "filosofía de gestión". Al hablar de estas técnicas, es necesario entender que el elemento medular de este pensamiento es el valor; mismo que es corroborado o no, por quien utiliza el producto o hace uso del servicio. Y tiene valor "cuando se expresa en términos de un producto específico -un buen servicio a menudo, ambos a la vez- que satisface las necesidades del consumidor a un precio concreto, en un momento determinado" (Womack y Jones, 2005 pp. 26).

Así, lean thinking es una metodología de gestión que surge a partir del lean manufacturing/lean production, la cual se define como "conjunto de técnicas desarrolladas por la compañía Toyota a partir del decenio de 1950 para mejorar y optimizar sus procesos operativos", Grupo Kaizen (2007), en Carrillo, Pons, Barrios y Puello (2010). Por su parte, Santos (2010) explica los principios lean de la siguiente forma:

- Una organización debe estar al servicio de todos sus grupos de interés (internos y externos).

- Implica considerar el valor agregado en todas sus formas.

- Los stakeholders de una organización ayudan al balance de ésta.

- Lean, implica empoderamiento de los clientes internos, y con ello, se logra un manejo participativo.

- Se debe incentivar de forma cotidiana el mejoramiento al interior de la organización, eso incluye la retroalimentación hacia el personal.

Aunque podría parecer que el utilizar el lean thinking es un canal forzado para transmitir la filosofía o la apertura al cambio, es una metodología que ha tenido éxito en muchas organizaciones; Rice y Taylor, (2003) en Hines y Lethbridge, (2008), mencionan que aún se tienen pocos casos de sistemas lean en instituciones académicas. En su mayoría, los análisis hacen referencia a algunas intervenciones enfocadas en la mejora continua y en la buscar calidad.

Algunos estudiosos se refieren a que los cambios deben ser eliminados junto con los desperdicios, y no optar por acercamientos holísticos e integrales al sistema. Ante esto, es necesario aplicar esta filosofía en todos los procesos administrativos (Araújo, 2011). Ahora bien, al realizar el vínculo entre lean thinking y universidades, las instituciones han logrado avances en 
la aplicación de ciertos procesos que ayudan a incrementar la forma en que se oferta el servicio educativo; pero este progreso se encamina a certificar algunos elementos (biblioteca, áreas específicas de la universidad, departamento, instalaciones, etc.), o para acreditar determinados programas de certificación. Sin embargo, al considerar los cambios profesionales, es prudente realizar modificaciones que involucren mayor agilidad en la preparación y la ejecución de programas académicos; todo ello, a través de una serie de dinámicas globales al interior de las IES (Carrillo, Pons, Barrios y Puello, 2010).

Por ello, diversas instituciones educativas crean sistemas de gestión de la calidad, los cuales se enfocan en prácticas esenciales como las Normas ISO, -especialmente ISO 9000, Sistemas de Acreditación de Programas e Instituciones, y el de los Premios -nacionales e internacionales- de Calidad (Carrillo, Pons, Barrios y Puello, 2010, pp. 6). Una vez que se habla del tema de innovación y lean thinking, como punto definitorio para tener un cambio -en múltiples aristasacadémico, es necesario entender otro tema, el de las resistencias al proceso de la innovación educativa.

Al hablar de resistencia, se parte del hecho de que ésta es: compleja y debe ser tomada como de suma importancia para el éxito del proceso innovador, en el sentido de que aparece como una oposición selectiva ante la posibilidad de una pérdida como producto del cambio (García, 2006), por eso no se debe obviar el hecho de que las IES sean capaces de mejorar el valor de sus procesos, uno de ellos, la eliminación de puestos, recursos, personal, entre otras acciones que suelen estar replicados o ser innecesarios (Hines y Lethbridge, 2008).

Sin embargo, a nivel académico es un factor más complicado, y esto se debe a que quizás la mayoría de las IES son organizaciones que están acostumbradas a no modificar sus estructuras, líneas de trabajo, políticas de gestión, entre otros aspectos que suelen impedir el crecimiento (Aaraújo, 2011). Por ello, es necesario considerar el potencial de quienes forman parte de la universidad, en particular los clientes internos; y es que, en ocasiones el conocimiento las competencias y las experiencias no se aprovechan al máximo (University of St Andrews Lean Team, 2011).

También existen otros problemas que limitan la aplicación de una logística lean para tener una ventaja competitiva. Bonal (1997) en García, (2006), informa sobre las ideologías que limitan el cambio y generan resistencia, a saber: de género y educativa, así como la inercia institucional; es decir, cada universidad propone costumbres y hábitos que pueden obstaculizar ciertos cambios a 
nivel educativo. En síntesis, se le considera como la integración que constituye una herramienta para tener una adecuada comprensión de la realidad (Guzmán, 2009).

Si se considera que un factor elemental en el proceso de la innovación es una preparación universitaria trans, multi e interdisciplinaria, es necesario considerar que, a nivel laboral, las distintas profesiones son grupos que se organizan y son autónomos al momento de desarrollar sus funciones, y éstos tienen algunos criterios y conocimientos basados en la formación académica (Castro y Chávez, 2012). En cuanto a la transdisciplinariedad, ésta constituye un paso importante para el sistema que actualmente rige a los niveles de trabajo e incluso a las ideologías científicas, esto, porque es parte al mismo tiempo de lo que es, y lo que va más allá de las disciplinas (Guzmán, 2009). Tiene un alcance que supera el marco educativo y académico, pues se logra posicionar como un pilar de cambios sociales, de ahí que sea parte fundamental de la innovación. Pero tampoco hay que olvidar que la interdisciplinariedad debe ser comprendida como un proceso (Peñuela, 2005), y por ello, hay que tenerla en cuenta en el marco de la innovación educativa.

\section{Metodología}

Para la elaboración de este análisis se opta por un método cualitativo; particularmente, con la herramienta de investigación documentada. Es decir, previo a la elaboración del modelo se realiza una consulta de la literatura correspondiente a los temas relacionados con la innovación educativa, la educación inter, trans y multidisciplinar, así como el pensamiento lean, la apertura al cambio, entre otros tópicos que se considera necesario abordar. A continuación, se presenta el modelo en la figura 1. 


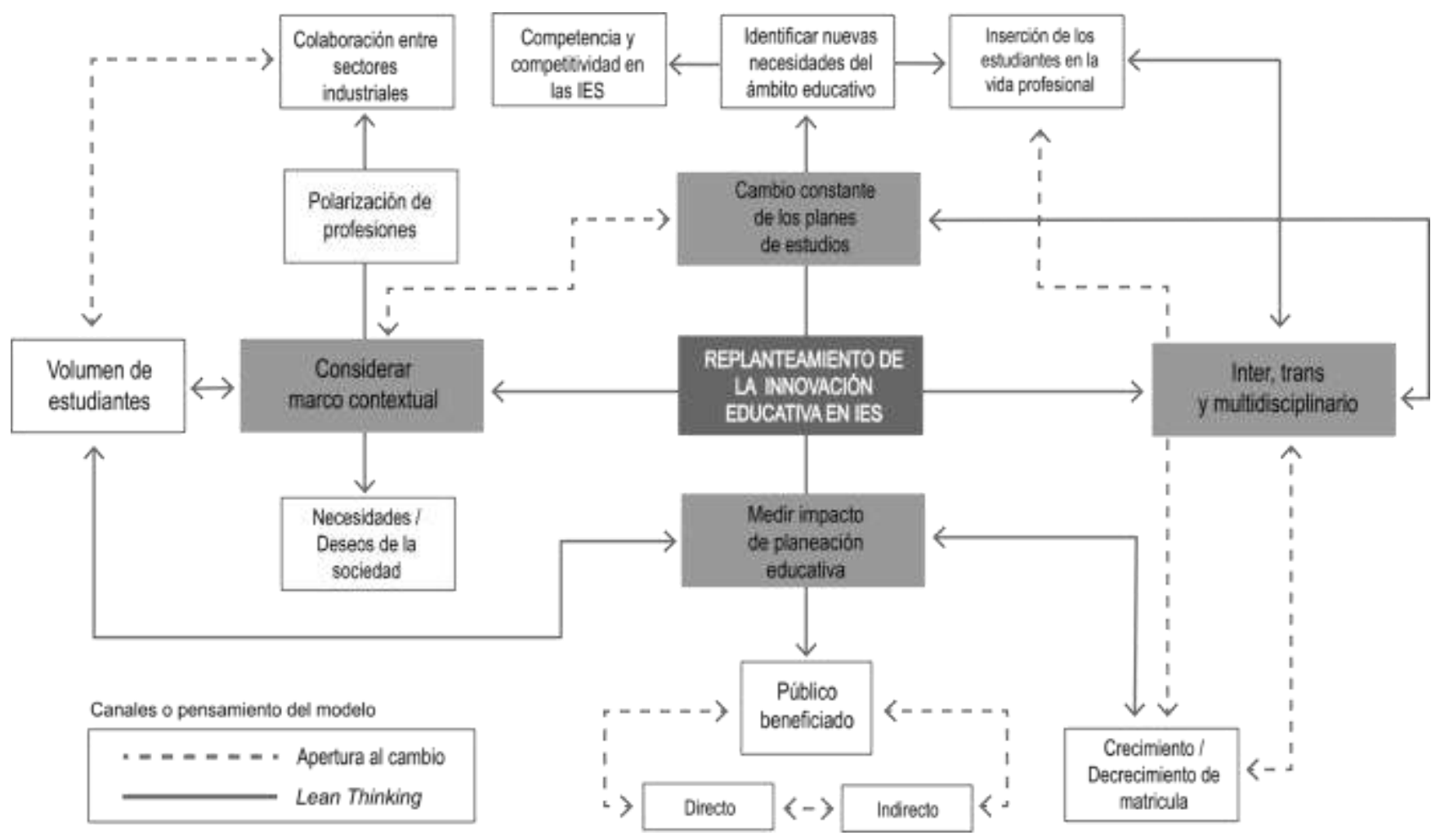

Figura 1. Modelo: "Replanteamiento de la educación en IES"

\section{Resultados}

Este modelo se sitúa en un contexto actual, en el cual dos elementos o factores son canales o medios para llevar acabo la innovación en las IES, además de propiciar constantemente una evolución en las organizaciones y ecosistemas de las universidades. La apertura al cambio y el lean Thiking conllevan a romper paradigmas, además de tener un pensamiento abierto y efectivo. El diagrama muestra cómo ciertos elementos están conectados por alguno de los dos canales seleccionados como medios; sin embargo, en la práctica no son excluyentes, pero se sugiere que sean predominantes para llevar a una mejor adaptación de las ideas expone el modelo.

Las cuatro ideas mencionadas alrededor de la casilla central, soportan el replanteamiento que menciona que la educación está en función de la necesidad en el cambio periódico de los planes de estudio; mismo que tiene como objetivo, identificar nuevas necesidades del cambio educativo. Estas ideas se les conoce -en este modelo- como secundarias. A raíz de dichas requisiciones, se da 
mayor competitividad y competencia entre las IES; lo cual se relaciona con la inserción que los estudiantes tengan o no, en la vida profesional y/o laboral.

Cabe recordar que no es obligación de las universidades que los estudiantes tengan o aseguren empleo una vez se gradúan o terminan su estudios o formación, y esto es lo que también debe ser replanteado en las aulas, e incluso en la propia ideología de las universidades, esto, sobre todo, porque en México el tema del desempleo es una apremiante que se presenta con mayor frecuencia. El modelo sugiere que la comunidad sea capaz de tener un amplio espectro en la colaboración con sectores fuera de la IES, pero, sobre todo, debe existir una idea conjunta académica, en la que se tenga la consigna de manifestar a los educandos que el aprendizaje también está en el marco contextual.

Todo esto, está ligado directamente a que la educación debe ser vista desde un aspecto en diversos ejes, entenderse como un prisma de diversas caras que tiene muchas lecturas, y que difícilmente se puede hablar de innovación educativa sino se trabaja la inter, multi y transdisciplinariedad. Esta segunda idea secundaria se ve directamente relacionada con el crecimiento o decrecimiento de la matrícula universitaria, y este es precisamente una de las formas en las que se puede tener una medición del impacto de la planeación educativa -tercera idea secundaria del modelo-.

Dicha cuantificación del éxito o fracaso se ve a través del público beneficiado a partir del cambio en la idea de pensar la universidad, y esto incluye al público directo e indirecto; pues a final de cuentas, lo que debe buscar esta flexibilidad es un mayor impacto en la sociedad, pues es el estudiante el que a través de una inmersión social, económica, artística y cultural en sus diversos cursos ha de entender cómo se suele desarrollar el mundo post universitario.

Pero todo esto no puede ser concebido si no se considera el marco contextual en el cual se desenvuelve el universo académico, y es que las necesidades y deseos de la sociedad son de gran importancia para que las IES logren sobrellevar los diversos planes de estudio, pues lo que en una época se consideraba esencial para la educación, ahora no lo puede ser. Una vez más, este factor se ve afectado directamente por el volumen de estudiantes que puede existir en una universidad, aspecto que evidentemente pertenece a una de las formas en las que se puede medir el impacto de planeación educativa.

Al considerar lo que en ocasiones pudiera necesitar la sociedad se llega a un punto de polarización de las profesiones, es decir, que puede haber generaciones universitarias que, debido 
a la transformación laboral u oportunidades de empleo decidan enfocarse en ciertos cursos exclusivamente; y esto no debiera ser común, pues la idea es que el estudiante tenga un abanico más amplio de posibilidades para forjar su aprendizaje, y no enfocarse en lo inmediato. Así pues, este modelo consigue que se entienda que las universidades deben ser entendidas desde otra perspectiva, y no sólo como elemento social educativo, por el contrario, debe comprenderse que estas casas de estudio son entes complejos que al momento de repensarse hay que analizar la mayor cantidad de agentes que intervienen en su concepción actual.

\section{Discusión}

La innovación educativa es parte de los cambios en que son generados por las necesidades de una nueva sociedad. La innovación cada día es más importante en los procesos que viven las empresas, organziaciones, dependencias gubernamentales, despachos, etc. y por supuesto, las IES. En latinoamérica, la innovación se ha apropiado de manera particular, pero despierta interés tanto académico como empresarial, pues se ha encontrado una necesidad de mejora tanto en la calidad educativa, como en una cultura de innovación en las diferentes actividades que se realizan (Mateos, Anderson y Rodríguez, 2012).

Se puede decir que en aras de generar perspectivas que favorezcan la innovación educativa, es necesario entender que las IES son sistemas que aprenden, que valoran críticamente los componentes de su entorno, que son sensibles a ellos y que con base en lo anterior se puede innovar en la práctica educativa y transformarla en su totalidad (Silas, 2006).

Actualmente, el ser humano se encuentra en un mundo que se caracteriza por el cambio constante e inesperado. Se está ante un paradigma en el que las IES han cambiado en aras de "entender" no sólo al estudiantado, sino a los padres de familia, profesores, administrativos, y demás grupos de interés que convergen en este espacio de conocimiento; sin embargo, esto no ha sido del todo sencillo, pues en ocasiones se llega a un punto de inflexión entre el cambio educativo y la tradición que ha caracterizado a las IES durante años.

Por ello, se cree necesario identificar cuáles pueden ser los elementos esenciales de una innovación al interior de las universidades, pues lo que se busca, de una u otra forma es que los estudiantes puedan vislumbrar el mundo en el que viven como algo que está en constante cambio, y lo que en alguna época se conocía como lo "estabelcido", ahora no lo es, y los procesos de innovación, en la mayoría de las ocasiones suelen ser la respuesta (Casas, 2005). Más allá de los 
primeros pasos en innovación vistos en el diseño curricular de planes de estudios en la IES (Morales, 2006), el replanteamiento de la innovación educativa es un campo fértil para sr trabajado de manera individual y colectiva.

\section{Conclusión}

El modelo multidireccional presentado en este trabajo desarrolla una serie de ideas claras sobre como replantear la innovación educativa. Las IES necesitan una reconfiguración en materia de innovación educativa, y son diversos los aspectos que deben ser considerados para lograrlo con éxito. Por otra parte, uno de los elementos de mayor importancia a nivel innovación, es la experiencia que puede servir como agente de cambio; sin embargo, esto tiene ciertas exigencias, y una de ellas es un cuerpo académico que posea nuevas y diversas formas de percibir la enseñanza y el aprendizaje; esto se puede lograr si ellos aportan diversas dimensiones profesionales (Tójar y Matas, 2005).

El trabajo aquí planteado apuestó por incluir la ideología de lean thinking; esto, porque es importante entender el progreso que tiene independientemente de los aspectos positivos o negativos al momento de ser implementado. Lo anterior, ayuda a entender los estatutos que pueden seguirse para que puedan ser aplicados al interior de las IES (Carrillo, Pons, Barrios y Puello, 2010).

A su vez, las universidades, que en teoría deben ser instituciones propicias al aprendizaje suelen rechazar la visión corporativa y se resisten a un cambio de visión en la que la mejora continua sea uno de los elementos característicos (Senge, 1990) en Lethbridge, (2010). Esto, en ocasiones limita su crecimiento, y lejos de convertirse en organizaciones capaces de innovar, y propiciar el cambio, se estacan en meros institutos que sólo buscan una retribución económica y se alejan de lo que, en teoría, fueron creados; espacios para la generación y transmisión de conocimientos.

Por ello se dice que la innovación mejora las condiciones de vida de las personas, y de ello se ocupan las instituciones que introducen cambios, en el sentido de aquello que está planeado; sumado a esto, cada universidad debe ocuparse de los diversos retos en su propio contexto; y es que las acciones estratégicas no solo disminuyen esas tendencias, sino pueden potenciar las diversas misiones universitarias (Rodríguez, 2015).

Por otra parte, no se puede negar que las IES tienen un rol primario en los procesos de innovación educativa en todos los niveles; y es que no solo se trata de proyectar el manejo de las 
IES en función de la innovación, sino entender todos los procesos desde la mayor cantidad de perspectivas de la integración de sus entornos, factores y procesos, en función de los procesos que hablan de innovación. Las IES son pieza clave para la generación de valor en múltiples factores por tres razones básicas:

1. La institución se concentra en un pensamiento social e intelectual en un contexto sociocultural.

2. Posibilidades reales de proyección formal de capital humano y cultural con vías a generar nuevos conocimientos, productos, alternativas y tecnologías.

3. Potencialidades de la universidad para la generación, promoción, generalización, evaluación y validación de la actividad innovadora y sus productos fundamentales.

No debe olvidarse que el sector del profesorado debe fomentar un cambio innovador, pues suelen promoverla mediante las estrategias y la aplicación de las metodologías del aprendizaje, mismas que fomentan una autogestión en el proceso de aprendizaje y creativo del alumnado; y no solo el uso de técnicas o medios, sino el desarrollo cognitivo o construcción del conocimiento y generación de sus habilidades profesionales (Rodríguez, 2015).

Además, es relevante tocar los términos: interdisciplinariedad, multidisciplinariedad o ámbitos transdisciplinares; para entender un sistema educativo y cómo se desarrollan estos términos de manera intrínseca en la innovación educativa. Las IES deben presentar una apertura a cambio y una filosofía eficiente para llevar más allá de la enseñanza y el aprendizaje múltiple, todo tipo de métodos, herramientas, mitologías y tecnologías para estar a la par de las tendencias venideras (Hernández, Castañeda y Del Castillo, 2000). Finalmente, el modelo multidireccional es un planetamiento que busca fomentar, mejorar y buscar un camino para llevar a una cultura y filosofía que toda institución u organización busca, que es el crecimiento humano, social y cultural de las sociedades (Expósito, 2018).

\section{Referencias}

Alatorre, E.P. (2006). Innovación y modalidades no convencionales. En G. Galindo, y C. Santos (Comp.), $1^{\mathrm{er}}$. Simposio Nacional de Investigación sobre la Innovación Educativa. Teoría, consideraciones éticas y prácticas, metodología y cambio educativo (169-178). Monterrey, México: Instituto Tecnológico y de Estudios Superiores de Monterrey.

Alemán, L., Gómez, M.G., Parada, M.E. y Sainz, P. (2012). Estrategias extracurriculares para la enseñanza de la innovación. Mateos, M. Anderson, y J.M. 
Alemán, L.Y. (2010). Liderazgo para la innovación en las cátedras de investigación del Tecnológico de Monterrey (Tesis de Maestría no publicada). Monterrey, México, Escuela de Graduados en Educación.

Álvarez R.V. y Lázaro M.Á. (2002). Calidad de las universidades y orientación universitaria. Málaga: Ediciones Aljibe.

Araújo, P. (2011). Universidades Lean: Contribución para la reflexión. Revista de la educación superior, 4(160), 135-154.

Beaver, D. de B. (2001). Reflections on scientific collaboration (and its study): past, present and future. Scientometrics, 5(23), 365-377.

Bonal, X. y Tomé, A, (1997). Construir la escuela coeducativa. La sensibilización del profesorado. Universidad Autónoma de Barcelona: Barcelona, España.

Caribbean Conference for Engineering and Technology (LACCEI'2010)

Carrillo, M.S., Pons, P.A., Barrios, M.A y Puello, P. (2010). Lean thinking metodología de gestión de mejoramiento en instituciones de educación superior. Latin American American and Caribbean Conference for Engineering and Technology. "Innovation and Development for the Americas". Arequipa, Perú.

Castro, Y. y Chávez, Y. (2012). La interdisciplinariedad en la formación profesional del analista de información: propuesta de competencias. Ciencias de la Información 43(2), 55-59.

Edgar, D., y Grant, K. (2009). Innovación en la práctica y practicando innovación. Documento de trabajo: Glasgow.

Ezpeleta, J. (2004). Innovaciones educativas. Reflexiones sobre los contextos en su implementación. Revista mexicana de investigación educativa. 21(9), 22-39.

Expósito, C. (2018). De la reflexión ideológica a la realidad pedagógica.

Revista de Investigación Apuntes Universitario, 8(2), 31-48.

Fernández, R. (2006). Condiciones para el surgimiento y aceptación de la innovación en educación. En G. Galindo, y C. Santos (Comp.), $1^{\mathrm{er}}$. Simposio Nacional de Investigación sobre la Innovación Educativa. Teoría, consideraciones éticas y prácticas, metodología y cambio educativo (17-29). Monterrey, México: Instituto Tecnológico y de Estudios Superiores de Monterrey.

Formichella, M.M. (2005). La evolución del concepto de innovación y su relación con el desarrollo. Recuperado de: www.unsch.edu.pe Consultado: Mayo-2018.

Fullan, M. G. (1996). Turning systemic thinking on its head. Phi Delta Kappan, 77(6), 420-423.

Galindo, y C. Santos (Comp.), $1^{\mathrm{er}}$. Simposio Nacional de Investigación sobre la Innovación Educativa. Teoría, consideraciones éticas y prácticas, metodología y cambio educativo (4357). Monterrey, México: Instituto Tecnológico y de Estudios Superiores de Monterrey.

García, M. (2006). Enfoques y voces sobre el cambio y la innovación educativa Memorias del Primer Simposio Nacional sobre Investigación en Innovación Educativa. ITESM. Monterrey, México.

Grupo Kaizen. (2007). Qué es el Lean Manufacturing. Recuperado de: http://www.grupokaizen.com/mck/ Consultado: Marzo-2018. 
Guzmán, M. (2009). Sistemas de organización del conocimiento y transdisciplinariedad: un acercamiento desde el enfoque de los niveles integrativos, Contribuciones cortas.

Havelock, R.G. y Huberman, A.M. (1980). Innovación y problemas de la educación: teoría y realidad en los países en desarrollo. Estudios y encuestas de educación comparada: París, Francia.

Hernández, L., Castañeda, A. Del Castillo, A. (2000). La transdisciplinariedad, una acción prioritaria para la educación superior a comienzos del tercer milenio. Revista Pedagogía Universitaria, (5)1.

Hines, P. y Lethbridge, S. (2008). New Development: Creating a Lean University. Public Money \& Management, 53-56.

Laurencio, A. y Farfán, P. (2016). La innovación educativa en el ámbito de la responsabilidad social Universitaria. Revista Cubana de Educación Superior, (2), 16-34.

Lašákováa, A., Bajzíkováa, L. y Dedze, I. (2017). Barriers and drivers of innovation in higher education: Case study-based evidence across ten European universities. International Journal of Educational Development, 55, 69-79.

Lethbridge, S. (2010). The Ethnography of Organizational Change Within a Lean University

Programme. Recuperado de:

https://www.liverpool.ac.uk/media/livacuk/schoolofmanagement/docs/abstracts/ethnograph y2010/Lethbridge.pdf, Consultado: Mayo-2018.

Livingston, K. y Assunção Flores, M. (2017) Trends in teacher education: a review of papers published in the European journal of teacher education over 40 years. European Journal of Teacher Education, 40(5), 551-560.

López, J. (2010). Sostenibilidad de la innovación en los centros escolares: sus bases institucionales. Revista de currículum y formación del profesorado, 14(1), 9-28.

Malander, N. (2014). Estrategias de aprendizaje y hábitos de estudio en el nivel superior: Diferencias según el año de cursado. Revista: Apuntes Universitarios 4(1), 9-22.

Marcelo, C. (1996). La innovación como formación. XI Congreso Nacional de Pedagogía. Innovación Pedagógica y Políticas Educativas: 43-86.

Mateos, A. Anderson, M. y Rodríguez, J.M. (2012). Nuevas formas de enseñar innovación. Signum: Salamanca.

Miranda, E. (2002). La supervisión escolar y el cambio educativo un modelo de supervisión para la transformación. Profesorado, revista de currículum y formación del profesorado, (6),1-2: 115.

Morales, J.J. (2006). Conocimiento e innovación para investigar en educación. En G. Galindo, y C. Santos (Comp.), $1^{\text {er }}$. Simposio Nacional de Investigación sobre la Innovación Educativa. Teoría, consideraciones éticas y prácticas, metodología y cambio educativo (141-151). Monterrey, México: Instituto Tecnológico y de Estudios Superiores de Monterrey.

Niño de Guzmán, J. C. (2015). La cultura organizacional en el contexto de la globalización. Revista Apuntes Universitarios, 5 (2), 19-40. 
Ortega P., Ramírez, M., Torres J., López, A., Yacapantli, A., Suírez L. y Ruiz B. (2007). Modelo de innovación educativa. Un marco para la formación y el desarrollo de una cultura de la innovación [versión electrónica. Revista Iberoamericana de Educación a Distancia (RIED), $10(1), 145-173$.

Patiño G., C.A. (2008). Centro Interamericano para el Desarrollo del Conocimiento en la Formación Profesional. Recuperado de: http://www.oit.org Consultado: Mayo-2018.

Pedroza F. y García, R y Briseño, B. (2005). Flexibilidad académica y curricular en las instituciones de educación superior. Miguel Ángel Porrúa: México.

Peñuela, A. (2005). La transdisciplinariedad más allá de los conceptos, la dialéctica Revista Andamios (1)2: 43-77.

Pineda, J.M. (1990). Resistencias a la innovación educativa. Studia pedagógica, 22, 45-53.

Pinto, J. (2006). Novas oportunidades. Revista Exame: 24-25.

Rivas, M. (2000). Innovación educativa. Teoría, procesos y estrategias. Síntesis: Madrid.

Rodríguez-Flores, M.E. (1997). La función directiva del escolar. Castillo: México.

Rodríguez, J. (2011). Nuevas formas de enseñar innovación Signum: Salamanca.

Rodríguez, A. (2011). Las principales políticas públicas hacia la educación universitaria. Globalización y neoliberalismo en la educación superior y otras ciencias sociales. Plaza y Valdez: México.

Rodríguez, A. (2015). Buenas prácticas como procesos y tendencias innovadoras en la educación superior. Revista panamericana de pedagogía saberes y quehaceres del pedagogo, 22, 5575.

Santiago, R. y Andía, (2016). Las percepciones de los directivos de centros escolares sobre el uso y el valor de las TIC para el cambio e innovación. Estudios sobre educación, 30, 145-174.

Santos, T. (2010). Análise da reacção dos colaboradores ao processo de mudança organizacional leanthinking. Um estudo de caso na empresa beta. Dissertação de Mestrado.

Silas, J.C. (2000). College curriculum and autopoiesis: A new framework of interpretation. Revista de la Escuela de Graduados en Educación, 1(1), 5-8.

Silas, J.C. (2001). The Systemic Character of the College Curriculum. Systemexico: The Mexican Journal of Systems Research. 2(1), 23-38.

Silas, J.C. (2006). La complejidad como un elemento para favorecer la innovación en educación. En G. Galindo, y C. Santos (Comp.), $1^{\mathrm{er}}$. Simposio Nacional de Investigación sobre la Innovación Educativa. Teoría, consideraciones éticas y prácticas, metodología y cambio educativo (31-42). Monterrey, México: Instituto Tecnológico y de Estudios Superiores de Monterrey.

Tejada, J. (1998). Los agentes de la innovación en los centros educativos: profesores, directivos y asesores. Aljibe: Málaga, España.

Téllez, D. (2011). Jóvenes niniy profesionistas titi: la estratificación letrada del desempleo. El Cotidiano, 169, 83-96. 
Tójar, J.C. y Terrón, A. (2005). El proceso de innovación educativa en la formación permanente del profesorado universitario: un estudio multicaso. Revista Española de Pedagogía, LXIII(232), 529-552.

Tourón, J. (2014). Diez tendencias que guían el futuro de la Educación. Recuperado de: www.javiertouron.es/diez-tendencias-que-guian-el-futuro-de/ Consultado: Mayo-2018.

University of St. Andrews, Lean Team. (2011). Becoming Lean: Pocket Guide. Escocia.

Valle, J. L. (2012). Los directores de CEIPS ante el espejo. El liderazgo educativo en un tiempo de cambio. Barcelona: Anthropos.

Womack, J. y Jones, D. (2005). Lean thinking. Cómo utilizar el pensamiento Lean para eliminar los despilfarros y crear valor en la empresa. Barcelona: Gestión 2000. 